\title{
EXTENDED CANONICAL SYSTEMS
}

\section{RAYMOND M. SMULLYAN ${ }^{1}$}

Let $L$ be a (finite) alphabet containing $K$ as a sub-alphabet; let $(S)$ be a system, like a Post canonical system $[1 ; 2]$, except that the production variables range over all strings in $K$ (rather than all strings in $L$, as in Post). The symbols in $L$ may, however, occur in the axioms and production statements of $(S)$. Such a system we call an extended canonical system; more specifically an $L-K$ system. We say that the system is in the alphabet $L$, but over the alphabet $K$. We have found such systems to be more wieldy than the Post systems; fewer axioms are usually required, and the axioms are usually shorter. ${ }^{2}$ It is easy to show the equivalence of representability in an extended canonical system to representability in a canonical system. It is well known that if $K$ contains only 1 symbol then not every recursively enumerable set of strings in $K$ is representable in a canonical system in the alphabet $K$; only the recursive sets can be so represented. We raise the problem: if $K$ contains only 1 symbol, is every r.e. set of strings in $K$ representable in some extended canonical system over $K$ ? We answer this question affirmatively.

To simplify our proof, somewhat, we shall appeal to Post's normal form theorem. ${ }^{3}$ We let $K$ be an alphabet containing just one symbol; call this symbol " 1 ". We shall identify a string of 1 's of length $n$ with the positive integer $n$. Let $A$ be a recursively enumerable set (of positive integers). Appealing to Post's normal form theorem, there is a normal canonical system (C) in the alphabet $\{1, b\}$ and a string $\sigma$ such that for every (positive) integer $n, n \in A$ iff $\sigma n$ is provable in (C). We let $K_{2}$ be the alphabet $\{1, b\}$ and we let $L$ be the 8 -symbol alphabet $\left\{1, b, N, C, T_{0}, P, Q,-\right\}$. We shall construct an $L-K$ system in which $A$ is represented. Along the way, we will have to represent certain relations of numbers (strings in $K$ ). For any $L-K$ system $(S)$, any string $\pi$ in $L$, and any relation $R\left(x_{1}, \cdots, x_{n}\right)$ of strings in $K$, the string $\pi$ is said to represent $R$ iff the following condition holds:

Received by the editors May 31, 1960.

1 This research was supported in part by a National Science Foundation Grant-inAid in Knot Theory and Metamathematics, Mathematics Department, Princeton University.

2 Extended canonical systems come very close to the elementary formal systems defined in [3].

It is not difficult to modify our proof so as to circumvent the normal form theorem. The main point of using the normal form theorem is to avoid the heavy metamathematical notation for general productions. 
for any strings $X_{1}, \cdots, X_{n}$ in $K, R\left(X_{1}, \cdots, X_{n}\right)$ holds iff the string $\pi X_{1}-X_{2}-\cdots-X_{n}$ is provable in $(S)$. [For a set $W$ of strings in $K, \pi$ represents $W$ in $(S)={ }_{d f}$ for every string $X$ in $K, X \in W$ iff $\pi X$ is provable in $(S)]$.

We assign Gödel numbers to all strings in $K_{2}$ as follows. We define $g(1)=1, g(b)=2$, and for any string $d_{n} d_{n-1} \cdots d_{1} d_{0}$ (where each $d_{i}$ is either 1 or $b$ ) we define $g\left(d_{n} d_{n-1} \cdots d_{1} d_{0}\right)$ to be the number $g\left(d_{0}\right)$ $+2 \cdot g\left(d_{1}\right)+4 \cdot g\left(d_{2}\right)+\cdots+2^{n} \cdot g\left(d_{n}\right)$. The Gödel correspondence $g$ is 1-1 from the set of all strings in $K_{2}$ onto all the positive integers. For any positive integer $i$ we let $E_{i}$ be that string in $K_{2}$ whose Gödel number is $i$. By $E_{i} E_{j}$ we mean $E_{i}$ followed by $E_{j}$, and we let $i * j$ be the Gödel number of $E_{i} E_{j}$. The function $x * y$ obeys the following conditions:

$$
\begin{aligned}
x * 1 & =2 x+1, \\
x * 2 & =2 x+2, \\
(x * y) * z & =x *(y * z) .
\end{aligned}
$$

Conversely, these 3 conditions uniquely determine the function $x * y$. We can thus represent the relation $x * y=z$ in the following $L-K$ system. [We are using $x, y, z, u, v, w$, as production variables. And, of course, these variables range over all strings in $K$.]

Axioms. N1

Productions.

$$
\begin{aligned}
& N x \rightarrow N x 1, \\
& N x \rightarrow C x-1-x x 1, \\
& N x \rightarrow C x-11-x x 11, \\
& C x-y-w, \quad C y-z-v, \quad C w-z-u \rightarrow C x-v-u .
\end{aligned}
$$

In the above $L-K$ system-call it $\left(S_{1}\right)$-the symbol " $N$ " represents the set of all positive integers (i.e. strings in $K$ ) and " $C$ " represents the relation $x * y=z$.

We let $T$ be the set of all strings provable in the normal canonical system (C), and we let $T_{0}$ be the corresponding set of Gödel numbers. We now wish to extend $\left(\mathrm{S}_{1}\right)$ to an $L-K$ system $\left(\mathrm{S}_{2}\right)$ in which $T_{0}$ is represented. We add to $\left(\mathrm{S}_{1}\right)$ the axiom $T_{0} n$, where $E_{n}$ is the one axiom of (C). And for each normal production $E_{i} x \rightarrow x E_{j}$ of (C), we add to $\left(\mathrm{S}_{1}\right)$ the production $C i-x-y, C x-j-z, T_{0} y \rightarrow T_{0} z$. In this system $\left(\mathrm{S}_{2}\right)$, " $T_{0}$ " represents the set of all numbers $n$ such that $E_{n}$ is provable in (C)-i.e. " $T_{0}$ " represents $T_{0}$.

The set $A$ is represented by some string $E_{d}$ in (C). Thus $A$ con- 
sists of the set of all $n$ such that $E_{d} n \in T$. The Gödel number of $E_{d}$ is, of course, $d$ and the Gödel number of $n$ (i.e. of a string of 1's of length $n)$ is $2^{n}-1$. Thus $A$ consists of all $n$ such that $d *\left(2^{n}-1\right) \in T_{0}$. We add to $\left(S_{2}\right)$ :

Axiom. $P 1-11$.

Production. $P x-y \rightarrow P x 1-y y$.

$P$ represents the set of all ordered pairs $(i, j)$ such that $2^{i}=j$.

Then we add:

Production. $P x-y 1, C d-y-z, T_{0} z \rightarrow Q x$.

In this system (S), " $Q$ " represents $A$.

\section{REFERENCES}

1. E. Post, Formal reductions of the general combinatorial decision problem, Amer. J. Math. vol. 65 (1943) pp. 192-215.

2. P. C. Rosenbloom, The elements of mathematical logic, New York, Dover Publications, Inc., 1950, Chapter IV.

3. R. M. Smullyan, Theory of formal systems, Annals of Mathematics Studies, no. 47, Princeton University Press, 1961.

Princeton University

\section{THE AXIOM FOR CONNECTED SETS}

\section{R. L. STANLEY}

1. Introduction. The basic motivation for this study was a desire to find a genuinely unified postulational principle which incorporated both the Axiom of Choice and the "axiom for sets," which latter means an appropriate analogue of the Aussonderungsaxiom to provide for the existence of sets. The possibility of thus uniting these two axiomatic principles has become especially interesting since adding the Axiom of Choice has been shown to be not only safe, ${ }^{1}$ but necessary as well. ${ }^{2}$ In particular, it was further hoped and expected that such a principle, when found, could be expressed naturally as a membership-equivalence statement-that is, essentially of the form

Presented to the Society, June 20, 1959 under the title The postulate for connected sets; received by the editors April 11, 1960 and, in revised form, May 31, 1960.

${ }^{1}$ Gödel showed [1] that for certain systems of set theory, adding the Axiom of Choice does not bring inconsistency.

${ }^{2}$ Mendelson showed [2] that the Axiom of Choice is independent of the other, usual axioms for set theory, hence is indispensable for developments which employ it essentially. 